\title{
Efeitos de um programa de jogos pré-desportivos nos aspectos psicobiológicos de idosas
}

\author{
The effects of a program of teaching games in psychobiological aspects of elderly women
}

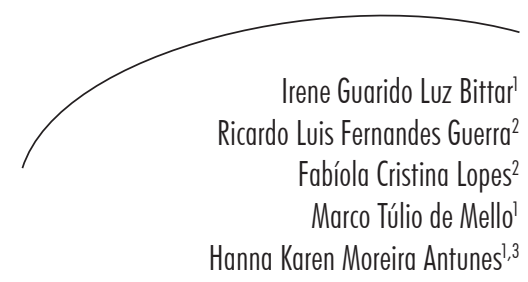

Resumo

Objetivo: Investigar o efeito de um programa de jogos pré-desportivos (JPD) nos aspectos psicobiológicos de idosas. Métodos: 53 voluntárias idosas foram distribuídas em dois grupos: grupo Ativo $(n=27)$ - submetido a um programa de JPD composto por atividades esportivas adaptadas, por seis meses, três vezes semanais, por uma hora; grupo Controle ( $\mathrm{n}=26$ ) - orientado a não modificar sua rotina, não se engajando em nenhum programa de atividade física sistematizada. A média ( \pm desvio-padrão) da idade, estatura, massa corporal, índice de massa corpórea foram: 67,91 $\pm 6,11 \mathrm{anos} ; 1,55 \pm 0,06 \mathrm{~cm}$; $80,68 \pm 8,45 \mathrm{~kg}$; e $33,41 \pm 2,62 \mathrm{~kg} / \mathrm{m}^{2}$, respectivamente. Antes e após o início do programa proposto, as voluntárias responderam a uma bateria psicobiológica composta por: miniexame do estado mental; escala de sonolência de Epworth; índice de qualidade de sono de Pittsburgh; SF-36; escala geriátrica de depressão; IDATE-Traço/Estado; escala de humor de Brunel; nível de atividade física habitual; e questionário de imagem corporal. Resultados: Após o término do período experimental, o grupo Ativo apresentou melhores respostas psicobiológicas de humor, representadas por menores escores de ansiedade, menores escores de depressão, melhor qualidade de vida com aumento da capacidade funcional e da vitalidade, além de melhora da imagem corporal, quando comparado ao grupo Controle. Conclusão: Os resultados sugerem que um programa de seis meses de JPD produz impacto positivo nos aspectos psicobiológicos de idosas.

\section{Abstract}

Objective: To investigate the effects of a program of teaching games (TG) in psychobiological aspects of elderly women. Methods: 53 female elderly volunteers were distributed in two groups: Active group ( $\mathrm{n}=27)$ participating of a TG program composed by adapted sporting activities for six months, three times a week, for one

\footnotetext{
Departamento de Psicobiologia, Centro de Estudos em Psicobiologia e Exercício. Universidade Federal de São Paulo. São Paulo, SP, Brasil.

2 Departamento de Ciências do Movimento Humano, Edifício Central. Universidade Federal de São Paulo. Santos, SP, Brasil.

3 Departamento de Biociências, Laboratório de Treinamento Físico Experimental. Universidade Federal de São Paulo. Santos, SP, Brasil.
}

Palavras-chave: Saúde do Idoso. Depressão. Cognição. Sono. Atividade Motora. Exercício. 
hour; Control group ( $n=26)$ was told not to change their routine and not to start any physical exercise regular program. The mean ( \pm standard-deviation) of height, weight, BMI were: $67.91 \pm 6.11$ years; $1.55 \pm 0.06 \mathrm{~cm} ; 80.68 \pm 8.45 \mathrm{~kg}$; and $33.41 \pm 2.62 \mathrm{~kg} /$ $\mathrm{m}^{2}$, respectively. Before and after of program proposed, the volunteers answered a psychobiology battery composed by: mini-mental state examination; Epworth sleepiness scale; Pittsburgh sleep quality index; SF-36; geriatric depression scale; STAI-trait/state; Brunel mood scale; habitual physical activity; and body shape questionnaire. Results: After the intervention period, the Active group showed better human psychobiological answers, represented by lower scores of anxiety and depression, better quality of life with increased functional capacity and vitality, and improved body image, when compared to the Control group. Conclusion: These results suggest that a 6-month TG program improves psychobiological aspects of elderly women.
Key words: Health of the Elderly. Depression. Cognition. Sleep. Motor Activity. Exercise.

\section{INTRODUÇÃO}

A população idosa vem aumentando o seu contingente em todo o mundo, e o Brasil vem se destacando desde a década de 1960 por ter um dos maiores índices de crescimento dessa população. ${ }^{1}$ Nos países desenvolvidos, o envelhecimento é acompanhado de melhorias da qualidade de vida, entretanto, no Brasil, o envelhecimento ocorre em outro contexto, caracterizado por desequilíbrios sociais e econômicos e por um serviço de saúde precário, que oferece baixa qualidade de vida para esse grupo etário emergente. ${ }^{2}$

$\mathrm{Na}$ população idosa brasileira, as mulheres são prevalentes em relação aos homens. ${ }^{1}$ Entre os problemas que afetam as mulheres idosas, queixas relacionadas aos aspectos psicobiológicos, isto é, aquelas que envolvem um contexto fisiológico capaz de interferir no comportamento ${ }^{3}$ têm se destacado. Entre os aspectos psicobiológicos mais relevantes podem-se citar aqueles envolvidos com alterações no padrão de sono; alterações no humor como depressão; alterações na função cognitiva, que em conjunto acabam refletindo na diminuição da sua autoestima e independência. ${ }^{4}$ Em suma, os idosos têm necessidades distintas das outras faixas etárias, e o aumento desse grupo populacional carece de implementações de políticas sociais e de saúde voltadas diretamente para esse público, evitando que percam a qualidade de vida e a autonomia em razão do desenvolvimento de incapacidades funcionais. ${ }^{2}$
A resposta a essas demandas exige atenção ao processo de envelhecimento. Novas estratégias para amenizar tais ocorrências podem ser implementadas. Nessa ótica, o uso de alternativas não medicamentosas, como exercícios físicos, vem a ser uma importante ferramenta para auxiliar essa população na busca de uma vida mais saudável e ativa, o que denota, em última instância, um comportamento adequado para retardar os possíveis efeitos negativos que advêm desse processo. A prática regular de exercícios físicos é uma estratégia preventiva primária, atrativa e eficaz para manter e melhorar o estado de saúde física e psíquica do idoso, apresentando efeitos benéficos diretos e indiretos para retardar as perdas funcionais do envelhecimento, reduzindo o risco de enfermidades e transtornos frequentes dessa faixa etária. ${ }^{5}$

Muitas atividades físicas dispensam recursos financeiros e também satisfazem necessidades particulares, tais como meditação, amizade, jogo e convivência, agindo sobre as emoções de forma a propiciar hábitos para uma melhor qualidade de vida. ${ }^{6}$ Dentre essas práticas, a vivência lúdicoesportiva pode ser encarada como meio para a construção de uma boa qualidade de vida, pois envolve o aluno em sua pluralidade, em seus aspectos históricos, culturais, físicos e sociais, quando a representação das ideias é exposta nas ações do jogo, de forma a acompanhar a subjetividade do conceito de qualidade de vida que considera os norteadores do contexto em que vive o indivíduo. 
Huizinga $a^{8}$ define o jogo como "uma atividade ou ocupação voluntária, exercida dentro de certos e determinados limites de tempo e de espaço, segundo regras livremente consentidas, acompanhado de um sentimento de tensão e de alegria e de uma consciência de ser diferente da 'vida cotidiana'". Nesse sentido, o estreitamento margeado pelas regras do jogo transforma os participantes em cúmplices, quando compartilham as regras, as representações, os espaços e conceitos, criando uma nova realidade, transferindo para a atmosfera lúdica a sua posição como indivíduos sociais. Dessa forma, o engrandecimento do contato social nessa condição pode vir a promover a redução da ansiedade, depressão, melhora na autoestima e na autoconfiança. ${ }^{?}$

Bunker \& Thoper $^{10}$ elaboraram uma proposta de modelo para jogos, o Teaching Games for Understanding (TGFU), em que o contexto e o cenário do jogo resultam no desenvolvimento da consciência tática e da tomada de decisão, favorecendo um aprendizado mais satisfatório. Nesse modelo, a tática é fundamentalmente trabalhada com as regras do jogo, para que a técnica seja sucessora de um bom desempenho com base nas tomadas de decisões mais apropriadas em cada momento. Essa proposta sugere a apreciação do jogo como ponto primário e permite que a iniciação esportiva seja trabalhada de forma pedagógica e abrangente.

Nesse sentido, a prática de jogos prédesportivos apresenta-se como estratégia interessante para alcançar uma boa qualidade de vida, pois suas adaptações podem ressaltar as características envolventes, motivadoras e inclusivas. Dessa forma, o componente lúdico de jogos pré-desportivos poderia atender às principais demandas provindas do processo do envelhecimento. Assim, o objetivo do presente estudo foi o de investigar os efeitos de um programa de jogos pré-desportivos nos aspectos psicobiológicos de idosas.

\section{METODOLOGIA}

Desenho do estudo

Trata-se de um ensaio clínico randomizado aplicado a uma amostragem de 53 idosas. O protocolo experimental foi realizado no período de fevereiro a agosto de 2008, nas dependências da UNIFESP - campus Baixada Santista, assim como as avaliações. O estudo foi divulgado via mídia social, televisão, panfletagem e jornal. As interessadas se dirigiram à Universidade para entrevista prévia, na qual os procedimentos do estudo foram esclarecidos e questões referentes aos critérios de inclusão e exclusão foram feitas para permitir o seu ingresso no estudo.

\section{Critérios de inclusão}

Foram incluídas no estudo mulheres sedentárias (que não realizassem nenhum tipo de atividade física de forma sistemática) com idade acima de 60 anos e índice de massa corporal (IMC) entre 30 e $35 \mathrm{~kg} / \mathrm{m}^{2}$.

\section{Critérios de exclusão}

Não participaram do estudo cardiopatas, tabagistas, portadoras de distúrbios do sono e de deficiências visuais ou auditivas, idosas que apresentassem dor musculoesquelética crônica ou dor aguda e intensa e as que consumiam bebida alcoólica mais de três vezes por semana.

\section{Procedimento ético}

Antes de participar, todas as voluntárias foram informadas quanto aos procedimentos e possíveis desconfortos e riscos envolvendo os processos de avaliação e de desenvolvimento experimental. Posteriormente, assinaram um Termo de Consentimento Livre e Esclarecido para a participação neste estudo. O estudo foi aprovado pelo Comitê de Ética em Pesquisa da 
Universidade Federal de São Paulo/ Hospital São Paulo (CEP 1714/08). Todos os procedimentos foram conduzidos de acordo com a Declaração de Helsinque (1975).

\section{Descrição da amostra e do protocolo experimental}

Participaram deste estudo 53 voluntárias idosas, obesas e sedentárias, com idade acima de 60 anos. O cálculo amostral foi realizado por meio do Power Analysis com auxílio do pacote estatístico Statistica versão 7.0. Nesse cálculo, o poder da amostra foi considerado em $80 \%$, e encontrado como adequado um $\mathrm{n}$ de 25 voluntários por grupo.

As voluntárias foram distribuídas randomicamente em dois grupos: a) grupo Controle: composto por 27 mulheres sedentárias que foram orientadas a não modificar suas atividades de rotina diária e a não se engajar em nenhum programa sistematizado que envolvesse exercício físico e/ou atividade física; b) grupo Ativo: composto por 26 mulheres sedentárias que foram submetidas a um protocolo de atividades físicas composto por jogos pré-desportivos por um período de seis meses, com atividades que foram realizadas três vezes por semana, durante 60 minutos por dia, em dias alternados. O grupo realizou a maioria das atividades motoras em jogos pré-desportivos em quadra ou campo, como atividades adaptadas ao voleibol, basquetebol, peteca e futebol, entre outras, em gradações de intensidade de leve a moderada. Como atividades complementares, em sessões de 20 minutos, foram ainda realizados: exercícios localizados para fortalecimento de braços, pernas, abdômen etc.; exercícios respiratórios para fortalecimento da musculatura respiratória em geral e prevenção de problemas respiratórios específicos; exercícios posturais (preventivos e de manutenção), principalmente para membros inferiores e coluna vertebral; exercícios de coordenação motora geral e específica e exercícios de equilíbrio.

Seguindo o protocolo experimental, em ambos os grupos, foram avaliados os seguintes quesitos: o perfil de humor, cognição, padrão de sono, imagem corporal, nível de atividade física habitual e qualidade de vida das voluntárias. Essa avaliação ocorreu em dois momentos distintos: 1) basal (antes de iniciar a intervenção); e 2) seis meses (seis meses após o início da intervenção).

Essas avaliações foram realizadas por meio de aplicação de questionários pelos pesquisadores, que foram previamente treinados para conhecerem a finalidade, a forma de entrevistar, o modo de contar os pontos e obter os resultados referentes a cada instrumento. Foi solicitado às voluntárias que respondessem os instrumentos honestamente, sendo garantido o total sigilo. Foi acordado que as voluntárias seriam informadas sobre a atualização dos resultados conhecidos pelos pesquisadores e que não haveria despesas pessoais para as participantes em qualquer fase do estudo, tampouco compensação financeira por sua participação.

Os questionários foram aplicados individualmente, em ambiente agradável, com luminosidade adequada e com o mínimo de ruído possível. A aplicação dos instrumentos foi feita em duas sessões de aproximadamente 45 minutos.

\section{Descrição dos instrumentos aplicados}

\section{Cognição}

Miniexame do Estado Mental (MEEM): teste de rastreio cognitivo, dividido em cinco subtestes: orientação, memória imediata, atenção e cálculo, evocação e linguagem. ${ }^{11}$

\section{Humor}

IDATE: é um questionário de autoavaliação dividido em duas partes: uma avalia a ansiedadetraço (referindo-se a aspectos de personalidade) e a segunda avalia a ansiedade-estado (referindose a aspectos sistêmicos do contexto). Cada uma dessas partes é composta de 20 afirmações. Ao 
responder o questionário, o indivíduo deve levar em consideração uma escala de quatro itens que variam de 1 a 4, sendo que ESTADO significa como o sujeito se sente no "momento" e TRAÇO como ele "geralmente se sente". O escore de cada parte varia de 20 a 80 pontos, sendo que os escores podem indicar um baixo grau de ansiedade (0-30), um grau mediano de ansiedade (31-49) e um grau elevado de ansiedade (maior ou igual a 50), quanto mais baixo se apresentarem os escores, menor será o grau de ansiedade. ${ }^{12}$

Escala Geriátrica de Depressão (EGD): este instrumento utilizado para a detecção de depressão em pessoas idosas oferece medidas válidas e confiáveis para a avaliação de transtornos depressivos. ${ }^{13}$

Escala de Humor de Brunel (BRUMS): desenvolvida para medir rapidamente o estado de humor. Consiste em uma lista de 24 adjetivos relacionados ao estado de humor. $\mathrm{Na}$ avaliação baseada neste instrumento, o avaliado deve responder como se sente em relação a cada um desses adjetivos, considerando uma escala tipo Likert de 0 a 4. Seis fatores de humor ou estados afetivos são medidos por esse instrumento: tensão, depressão, raiva, vigor, fadiga e confusão, que, combinados, caracterizam o distúrbio total de humor (DTH). É esperado nesse teste que os valores encontrados para a dimensão vigor sejam maiores que os valores apresentados nas outras dimensões, o que denotaria um perfil de humor em forma de iceberg. ${ }^{14}$

\section{Qualidade de vida}

SF-36 - Pesquisa em saúde: questionário genérico de avaliação de qualidade de vida Medical Outcomes Study SF-36. Esse questionário tem o propósito de avaliar, de forma genérica, a qualidade de vida, por ser de fácil e rápida administração e compreensão. Trata-se de um instrumento multidimensional composto por 36 itens, avaliando em oito dimensões: a) dez itens relacionados com a capacidade funcional (CF); b) quatro itens sobre aspectos físicos (AF); c) dois itens sobre dor; d) cinco itens relacionados ao estado geral de saúde (EGS); e) quatro itens sobre vitalidade (VI); f) dois itens sobre limitação por aspectos sociais (LAS); g) três itens sobre limitação por aspectos emocionais (LAE); h) cinco itens relacionados a saúde mental (SM) e ainda uma questão de avaliação comparativa entre as condições de saúde atual e as de um ano atrás.

Para avaliar os resultados, é determinado um escore para cada uma das questões que, posteriormente, são transformadas em escala de 0 (zero) a 100 (cem). "Zero" corresponde a um pior estado de saúde e "cem" a um melhor estado. Cada uma das dimensões é analisada separadamente. ${ }^{15}$

\section{Sono}

Índice de Qualidade de Sono de Pittsburgh (PSQI): questionário que avalia a qualidade de sono. Escores menores que 5 pontos são indicativos de normalidade. Já valores maiores ou iguais a 5 pontos indicam qualidade de sono pobre. $^{16}$

Escala de Sonolência de Epworth (ESS): questionário que avalia o nível de sonolência diurna. Os escores variam de 0 a 24 pontos. Escores até 10 pontos indicam normalidade, enquanto valores acima de 10 pontos sugerem sonolência diurna excessiva. ${ }^{16}$

Questionário de Cronotipo: utilizado para a caracterização da matutinidade/vespertinidade. Ele é amplamente utilizado nos estudos do ciclo vigília-sono, sendo composto pelos seguintes critérios: horários preferenciais de acordar e dormir; horários de maior disposição para atividades físicas e intelectuais; grau de dificuldade com que a pessoa executa determinadas tarefas em determinados horários e a autoclassificação da pessoa em um dos cinco tipos de cronotipo (matutino, moderadamente matutino, indiferente, moderadamente vespertino e vespertino). ${ }^{17}$ 


\section{Padrão social}

Questionário de Padrão Social - Abipeme: classificação que determina nível socioeconômico por intermédio da atribuição de pesos a um conjunto de itens de conforto doméstico, além de nível de escolaridade do chefe da família. A classificação socioeconômica da população é apresentada por meio de cinco principais classes, denominadas A, B, C, D e E..$^{18}$

\section{Nível de atividade física}

Nível de Atividade Física Habitual (NAFH): questionário que avalia o nível de atividade física habitual observando três dimensões: atividade de trabalho, esporte e lazer. Os índices derivam dessas dimensões. ${ }^{19}$

\section{Imagem corporal}

Questionário de Imagem Corporal (BSQ): avalia a imagem corporal, traduzido e validado para a população brasileira. O escore varia de 34 a 204 pontos. A classificação dos escores indica os diferentes graus de distorção da imagem corporal: ausência de distorção $(\leq 80)$, leve distorção (81-110), moderada distorção (111-140), grave distorção $(\geq 141) .{ }^{20}$
Análise estatística

Inicialmente foi aplicado o teste Kolmogorov-Smirnov para determinação da curva de normalidade. Para descrição dos dados foi utilizado o recurso de distribuição de frequências relativas e análise descritiva, quando necessário. Para a comparação entre os grupos e os tempos foi utilizada uma ANOVA para medidas repetidas, seguida de Duncan como post-hoc. Para analisar os dados nominais, foi utilizado o teste exato de Fisher. O nível de significância adotado em todas as análises foi de $\mathrm{p} \leq 0,05$, e os dados estão apresentados em média \pm desvio-padrão ou em porcentagem, quando necessário. A análise estatística foi conduzida com o auxílio do software Statistica Statsoft, versão 7.0.

\section{RESULTADOS}

$\mathrm{Na}$ tabela 1 são apresentados os dados descritivos das características iniciais da amostra. Quando os grupos foram comparados não foram encontradas diferenças significativas, demonstrando a homogeneidade da amostra. Para melhor elucidação dos dados, optou-se em demonstrar a tabela com dados de todas as voluntárias, seguida pelos grupos experimentais. 
Tabela 1 - Análise descritiva das características da amostra. São Paulo, SP, 2008.

\begin{tabular}{|c|c|c|c|c|}
\hline & Variáveis & n (53) & Ativo (27) & Controle (26) \\
\hline \multicolumn{2}{|c|}{ Idade (anos) } & $67,90 \pm 6,11$ & $68,41 \pm 6,82$ & $67,38 \pm 5,36$ \\
\hline \multicolumn{2}{|c|}{ Massa corporal total (kg) } & $80,68 \pm 8,45$ & $80,35 \pm 8,47$ & $81,02 \pm 8,57$ \\
\hline \multicolumn{2}{|c|}{ Estatura (m) } & $1,55 \pm 0,06$ & $1,57 \pm 0,07$ & $1,54 \pm 0,05$ \\
\hline \multicolumn{2}{|c|}{$\operatorname{IMC}\left(\mathrm{kg} / \mathrm{m}^{2}\right)$} & $33,41 \pm 2,62$ & $32,74 \pm 2,31$ & $34,09 \pm 2,80$ \\
\hline \multirow{5}{*}{ 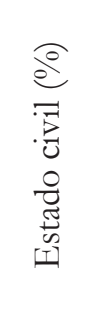 } & Viúva & $28,30(15)$ & $29,63(8)$ & $26,92(7)$ \\
\hline & Casada & $43,40(23)$ & $51,85(14)$ & $34,62(9)$ \\
\hline & Solteira & $9,43(5)$ & $7,41(2)$ & $11,54(3)$ \\
\hline & Separada & $11,32(6)$ & $11,11(3)$ & $11,54(3)$ \\
\hline & Divorciada & $7,55(4)$ & 0 & $15,38(4)$ \\
\hline \multirow{6}{*}{ 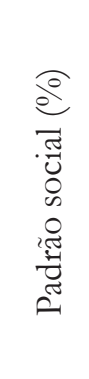 } & A1 & $7,55(4)$ & $3,70(1)$ & $11,54(3)$ \\
\hline & A2 & $9,43(5)$ & $11,11(3)$ & $7,69(2)$ \\
\hline & B1 & $16,98(9)$ & $25,93(7)$ & $7,69(2)$ \\
\hline & B2 & $32,08(17)$ & $33,33(9)$ & $30,77(8)$ \\
\hline & $\mathrm{C}$ & $30,19(16)$ & $25,93(7)$ & $34,62(9)$ \\
\hline & $\mathrm{D}$ & $3,77(2)$ & 0 & $7,69(2)$ \\
\hline \multirow{5}{*}{ 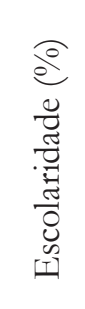 } & Analfabeta & $9,43(5)$ & $7,41(2)$ & $11,54(3)$ \\
\hline & Primário completo & $26,42(14)$ & $29,63(8)$ & $23,08(6)$ \\
\hline & Ginásio completo & $15,09(8)$ & $11,11(3)$ & $19,23(5)$ \\
\hline & Colegial completo & $26,42(14)$ & $25,93(7)$ & $26,92(7)$ \\
\hline & Superior completo & 22,64 (12) & $25,93(7)$ & $19,23(5)$ \\
\hline
\end{tabular}

Dados apresentados em média \pm desvio-padrão ou porcentagem, quando necessário. O número entre parênteses refere-se ao número de voluntários em cada item ou grupo. Não foram observadas diferenças significativas nas variáveis estudadas quando os grupos foram comparados.

Na tabela 2 são apresentados os resultados referentes às variáveis de ansiedade, perfil de humor, qualidade de vida, imagem corporal e sono considerando os grupos e os tempos. As análises dos escores do estado de ansiedade revelaram que no grupo Ativo após seis meses de intervenção houve diminuição dos escores $(\mathrm{p}=0,04)$. No instrumento que avaliou o perfil de humor (BRUMS), na dimensão depressão, o grupo Ativo apresentou redução dos escores quando comparado ao momento basal $(\mathrm{p}=0,02)$, enquanto que o grupo Controle apresentou aumento dos escores da dimensão fadiga $(\mathrm{p}=0,02)$ no mesmo tipo de análise. $\mathrm{Na}$ comparação intergrupo, o grupo Ativo apresentou menores escores após o período de intervenção nas dimensões depressão $(p=0,05)$, fadiga $(p=0,02)$ e DTH $(\mathrm{p}=0,04)$ sugerindo um melhor perfil de humor. Em relação à qualidade de vida, na comparação intragrupo, observou-se que o grupo Ativo apresentou maiores escores em relação à média das dimensões, capacidade funcional $\mathrm{e}$ vitalidade ( $\mathrm{p}=0,03$ para todos). Já na comparação intergrupos, observou-se que o grupo Ativo 
apresentou maiores escores após o período de intervenção nas dimensões dor $(\mathrm{p}=0,04)$, aspecto social $(\mathrm{p}=0,03)$ e na média das dimensões $(\mathrm{p}=0,05)$, permitindo sugerir uma melhora na qualidade de vida deste grupo. Outro ponto favorável ao grupo Ativo refere-se aos menores escores observados no BSQ após o término do período de intervenção $(\mathrm{p}=0,01)$, indicando melhora na imagem corporal. Em relação ao sono, observou-se que o grupo Controle apresentou maiores escores no PSQI e no ESS indicando menor qualidade de sono e sonolência excessiva diurna, respectivamente. Nas outras análises não foram encontradas diferenças significativas.

Tabela 2 - Variáveis de humor, qualidade de vida, imagem corporal e sono. São Paulo, SP, 2008.

\begin{tabular}{|c|c|c|c|c|c|}
\hline \multicolumn{2}{|r|}{ Variáveis } & \multicolumn{2}{|c|}{ Ativo (27) } & \multicolumn{2}{|c|}{ Controle (26) } \\
\hline & & Basal & 6 meses & Basal & 6 meses \\
\hline \multirow{2}{*}{ 莺 } & Traço & $35,11 \pm 9,38$ & $32,31 \pm 8,40$ & $41,08 \pm 11,83$ & $38,63 \pm 12,12$ \\
\hline & Estado & $32,81 \pm 9,90$ & $29,08 \pm 6,73^{*}$ & $33,38 \pm 7,10$ & $30,68 \pm 5,79$ \\
\hline \multirow{7}{*}{$\sum_{\infty}^{\infty}$} & Tensão-ansiedade & $2,37 \pm 2,45$ & $1,33 \pm 1,84$ & $2,27 \pm 3,33$ & $2,37 \pm 2,56$ \\
\hline & Depressão & $1,19 \pm 0,73$ & $0,23 \pm 0,82 *$ & $1,50 \pm 2,90$ & $2,00 \pm 3,33 \#$ \\
\hline & Raiva-hostilidade & $0,52 \pm 1,16$ & $0,31 \pm 0,55$ & $0,38 \pm 1,39$ & $1,16 \pm 2,83$ \\
\hline & Vigor & $10,78 \pm 2,61$ & $11,38 \pm 3,63$ & $10,27 \pm 3,24$ & $10,89 \pm 3,02$ \\
\hline & Fadiga & $1,33 \pm 1,94$ & $0,77 \pm 1,53$ & $1,50 \pm 2,21$ & $3,05 \pm 2,76^{*} \#$ \\
\hline & Confusão mental & $0,89 \pm 1,48$ & $0,73 \pm 1,51$ & $1,58 \pm 2,27$ & $1,58 \pm 2,87$ \\
\hline & DTH & $-4,44 \pm 8,37$ & $-8,23 \pm 5,60$ & $-3,00 \pm 10,55$ & $-0,74 \pm 13,94 \#$ \\
\hline \multirow{9}{*}{$\begin{array}{l}\infty \\
\infty \\
i \\
\omega\end{array}$} & Média das dimensões & $75,87 \pm 15,04$ & $84,12 \pm 10,43^{*}$ & $70,44 \pm 16,36$ & $73,95 \pm 15,39 \#$ \\
\hline & Capacidade funcional & $75,00 \pm 15,63$ & $82,12 \pm 15,44^{*}$ & $65,77 \pm 18,26$ & $71,58 \pm 18,49$ \\
\hline & Aspectos físicos & $87,96 \pm 24,39$ & $88,85 \pm 24,38$ & $70,19 \pm 34,65$ & $75,00 \pm 37,27$ \\
\hline & Dor & $64,15 \pm 27,15$ & $74,23 \pm 23,67$ & $58,00 \pm 23,42$ & $56,79 \pm 16,14 \#$ \\
\hline & Escala geral de saúde & $77,67 \pm 21,76$ & $85,42 \pm 17,20$ & $75,15 \pm 19,02$ & $76,68 \pm 21,73$ \\
\hline & Vitalidade & $69,63 \pm 21,26$ & $80,38 \pm 14,35^{*}$ & $67,50 \pm 25,11$ & $70,00 \pm 25,11$ \\
\hline & Aspecto social & $82,41 \pm 20,59$ & $92,79 \pm 12,83$ & $78,65 \pm 21,09$ & $78,94 \pm 22,46 \#$ \\
\hline & Aspecto emocional & $72,84 \pm 35,85$ & $84,61 \pm 31,60$ & $75,64 \pm 35,97$ & $85,96 \pm 25,62$ \\
\hline & Saúde mental & $77,63 \pm 19,29$ & $81,08 \pm 16,42$ & $69,54 \pm 28,76$ & $74,11 \pm 24,05$ \\
\hline \multicolumn{2}{|c|}{ Escore BSQ } & $78,44 \pm 27,27$ & $67,92 \pm 24,63^{*}$ & $91,23 \pm 36,41$ & $77,53 \pm 30,14$ \\
\hline \multicolumn{2}{|c|}{ Escala geriátrica de depressão } & $5,63 \pm 4,51$ & $5,65 \pm 4,60$ & $8,92 \pm 6,64$ & $8,21 \pm 6,73$ \\
\hline \multirow{2}{*}{ Sono } & PSQI & $6,23 \pm 3,71$ & $5,19 \pm 3,38$ & $6,58 \pm 4,32$ & $5,21 \pm 2,68 \#$ \\
\hline & ESS & $5,56 \pm 3,79$ & $6,65 \pm 3,78$ & $7,31 \pm 5,32$ & $9,47 \pm 5,55^{*} \#$ \\
\hline
\end{tabular}

ANOVA para medidas repetidas com post hoc Duncan test. *Diferente do basal intragrupo; \#diferença intergrupo no mesmo tempo, resultados diferentes para $\mathrm{p} \leq 0,05$. Dados apresentados em média \pm desvio-padrão. Imagem Corporal- escore do questionário BSQ; qualidade de sonoíndice de qualidade de sono de Pittsburgh; sonolência- escala de sonolência de Epworth. 
$\mathrm{Na}$ tabela 3 apresenta-se o resultado do screening cognitivo realizado por meio do MEEM. Foram encontradas diferenças apenas entre os grupos no item atenção e cálculo após o período de intervenção, onde o grupo Ativo apresentou maiores escores $(\mathrm{p}=0,05)$ quando comparado ao grupo Controle. Nas outras análises não foram encontradas diferenças significativas.

Tabela 3 - Variáveis cognitivas. São Paulo, SP, 2008.

\begin{tabular}{|c|c|c|c|c|c|}
\hline & \multirow{2}{*}{ Variáveis } & \multicolumn{2}{|c|}{ Ativo (27) } & \multicolumn{2}{|c|}{ Controle (26) } \\
\hline & & Basal & 6 meses & Basal & 6 meses \\
\hline \multirow{6}{*}{ 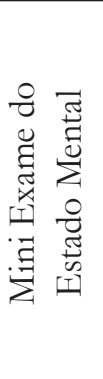 } & Orientação & $9,48 \pm 0,58$ & $9,38 \pm 0,98$ & $9,23 \pm 1,03$ & $9,53 \pm 1,17$ \\
\hline & Memória imediata & $3,00 \pm 0$ & $3,00 \pm 0$ & $2,92 \pm 0,39$ & $3,00 \pm 0$ \\
\hline & Atenção e cálculo & $3,37 \pm 1,62$ & $3,92 \pm 1,52$ & $2,46 \pm 2,02$ & $2,79 \pm 1,55 \#$ \\
\hline & Evocação & $1,93 \pm 1,07$ & $2,27 \pm 0,87$ & $1,77 \pm 0,91$ & $2,00 \pm 1,00$ \\
\hline & Linguagem & $8,64 \pm 0,53$ & $8,36 \pm 0,87$ & $8,53 \pm 0,73$ & $8,54 \pm 0,59$ \\
\hline & Escore total & $26,42 \pm 2,28$ & $26,93 \pm 2,35$ & $25,80 \pm 3,19$ & $25,75 \pm 2,47$ \\
\hline
\end{tabular}

ANOVA para medidas repetidas com post hoc Duncan test. \#Diferença intergrupo no mesmo tempo, resultados diferentes para $\mathrm{p} \leq 0,05$. Dados apresentados em média \pm desvio-padrão.

$\mathrm{Na}$ tabela 4 apresentam-se os resultados do nível de atividade física habitual. Quando os grupos e os tempos foram comparados, observouse que na média das dimensões, bem como nas dimensões esportivo e lazer, após o período de intervenção, o grupo Controle apresentou menores escores $(\mathrm{p}=0,01, \mathrm{p}=0,01$ e $\mathrm{p}=0,04$, respectivamente) quando comparado com o grupo Ativo. Além disso, o grupo Ativo aumentou os escores na dimensão lazer quando comparado a condição basal $(p=0,04)$. Nas outras análises não foram encontradas diferenças significativas.

Tabela 4 - Nível de atividade física habitual. São Paulo, SP, 2008.

\begin{tabular}{|c|c|c|c|c|c|}
\hline & \multirow{2}{*}{ Variáveis } & \multicolumn{2}{|c|}{ Ativo (27) } & \multicolumn{2}{|c|}{ Controle (26) } \\
\hline & & Basal & 6 meses & Basal & 6 meses \\
\hline \multirow{5}{*}{ 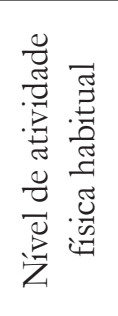 } & Ocupacional & $3,00 \pm 0,51$ & $2,97 \pm 0,37$ & $2,80 \pm 0,82$ & $2,96 \pm 0,89$ \\
\hline & Esportivo & $2,20 \pm 0,45$ & $2,18 \pm 0,65$ & $1,86 \pm 0,44$ & $1,70 \pm 0,51 \#$ \\
\hline & Lazer & $2,77 \pm 0,56$ & $3,09 \pm 0,52^{*}$ & $2,63 \pm 0,72$ & $2,61 \pm 0,63 \#$ \\
\hline & Absoluto & $7,97 \pm 0,99$ & $8,17 \pm 1,42$ & $7,27 \pm 1,45$ & $7,27 \pm 1,12$ \\
\hline & Média das dimensões & $2,66 \pm 0,33$ & $2,97 \pm 1,01$ & $2,42 \pm 0,48$ & $2,42 \pm 0,37 \#$ \\
\hline
\end{tabular}

ANOVA para medidas repetidas com post boc Duncan test. *Diferente do basal para o mesmo grupo; \#diferença intergrupos no mesmo tempo, resultados diferentes para $\mathrm{p} \leq 0,05$. Dados apresentados em média \pm desvio-padrão. 
Na tabela 5 é apresentado o resultado da média do cronotipo e a sua respectiva distribuição de frequências relativas, tanto para o grupo Ativo quanto para o grupo Controle a maior parte da amostra encontra-se na classificação de matutino moderado e apenas o grupo Controle apresenta classificação indiferente e vespertino moderado.

Tabela 5 - Cronotipo. São Paulo, SP, 2008.

\begin{tabular}{|c|c|c|c|}
\hline & Variáveis & Ativo (27) & Controle (26) \\
\hline \multicolumn{2}{|c|}{ Média do escore } & $66,78 \pm 5,34$ & $63,32 \pm 9,58$ \\
\hline \multirow{5}{*}{ 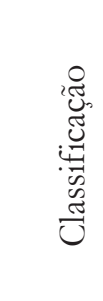 } & Matutino extremo & $37,04(10)$ & $26,92(7)$ \\
\hline & Matutino moderado & $62,96(17)$ & $53,85(14)$ \\
\hline & Indiferente & 0 & $15,38(4)^{*}$ \\
\hline & Vespertino moderado & 0 & $3,85(1)$ \\
\hline & Vespertino extremo & 0 & 0 \\
\hline
\end{tabular}

Distribuição de frequências relativas, dados apresentados em porcentagem. O número entre parênteses representa o número de voluntários total em cada classificação do cronotipo. *Exato de Fisher (amostras independentes), p $\leq 0,05$.

\section{DISCUSSÃO}

Os dados sistematizados neste estudo sugerem que o programa de jogos pré-desportivos utilizado foi efetivo em promover modificações benéficas na qualidade de vida, no humor e na qualidade de sono das voluntárias. Por outro lado, não foram alcançadas modificações na cognição, o que pode ser medido pelo escore final do instrumento miniexame do estado mental. Somente na dimensão atenção e cálculo foi observada uma melhora.

Em parte, esse resultado pode ser atribuído ao tipo de intervenção adotado. Possivelmente, por se tratar de jogos pré-desportivos, que apresentam em sua essência um caráter lúdico e a intensidade das atividades ser relativamente baixa, tal atividade não foi suficiente para promover modificações benéficas na cognição. Angevaren et al. ${ }^{21}$ defendem que a intensidade do exercício físico tem um importante papel na melhoria da função cognitiva. Segundo os autores, atividades de intensidade mais elevada promovem um maior impacto no sistema cardiovascular, resultando em aumento do fluxo sanguíneo cerebral e aumentando o transporte de oxigênio para o cérebro. Antunes et al. ${ }^{22,23}$ sugerem que exercícios realizados com predominância no metabolismo aeróbio (LV1) são efetivos em melhorar o desempenho em testes cognitivos de idosos. A partir dessa ótica, é possível sugerir que o protocolo utilizado neste estudo não atingiu essa intensidade, justificando os resultados encontrados.

Essa especulação encontra reforço em Angevaren et al., ${ }^{21}$ de acordo com quem os efeitos benéficos do exercício físico na função cognitiva poderiam ser explicados por mecanismos cardiovasculares, tais como: aumento do fluxo sanguíneo cerebral, ${ }^{24}$ influência do exercício aeróbio nos componentes do condicionamento cardiovascular ${ }^{25}$ e diminuição da viscosidade do sangue ${ }^{26}$ que por sua vez podem melhorar a performance cognitiva. ${ }^{26,27} \mathrm{O}$ trabalho de Barnes et al. ${ }^{28}$ teve como objetivo determinar se o condicionamento cardiorrespiratório está associado com a manutenção da função cognitiva, para isso, os autores verificaram maior vasodilatação cerebral em idosos com maior capacidade aeróbia, medida pelo índice de volume de oxigênio máximo. 
Embora ainda existam dúvidas, ${ }^{29}$ de forma geral, tem sido amplamente aceito na literatura ${ }^{23-28}$ que a prática regular de exercício físico, particularmente aquele executado com predominância no metabolismo aeróbio, apresenta influência positiva na cognição. É possível que as disparidades encontradas na literatura se devam aos mecanismos propostos para justificar esse efeito, talvez o papel do condicionamento cardiovascular seja mais amplo do que o especulado atualmente, uma vez que o exercício aeróbio além de melhorar aspectos cardiovasculares, atua na liberação de estimuladores neurogênicos que agem na manutenção da função cerebral e na promoção da plasticidade neural, capazes de modular a cognição, como o BDNF (brain-derived neurotrophic factor), e sua prática associa-se com o aumento do volume do hipocampo cerebral. ${ }^{30} \mathrm{E}$ importante mencionar que o BDNF é um membro da família dos neurotróficos que mantém a saúde e funcionamento dos neurônios primários glutamatérgicos. Os mecanismos que induzem o BNDF, como o exercício físico, melhoram a aprendizagem, sendo que a manutenção do nível deste neurotrófico é importante para a efetiva função neural e longevidade. ${ }^{31}$

Tendo em vista a manutenção da função cognitiva na idosa, a utilização de um programa de jogos pré-desportivos representa uma alternativa viável, pois, em função da monotonia, alguns programas clássicos de treinamento não alcançam os resultados esperados, ${ }^{32}$ e a variação das atividades desenvolvidas durante os jogos pré-desportivos, concomitantemente aos diversos estímulos cognitivos durante esta atividade, poderia intervir mais efetivamente, em função do seu nível de intensidade mais baixo. O caráter lúdico e o nível baixo de intensidade do programa representou um aumento nas atividades de lazer, como observado nos resultados para NAFH das participantes. Outro ponto importante é o fato de não se observar alterações significativas em relação aos escores indicativos de depressão medidos pela EGD. De acordo com Antunes et al., ${ }^{33}$ modificações nesta variável são decorrentes de treinamentos aeróbios. Isso se deve à promoção de lipólise resultante desse tipo de treinamento, que aumenta a quantidade de ácidos graxos livres, o que por sua vez desloca o triptofano da albumina, aumentando a fração livre desse aminoácido. Essa fração aumentada concorre com os aminoácidos de cadeia ramificada na passagem pela barreira hematoencefálica, considerando também que a musculatura que está sendo exercitada utiliza-se desses aminoácidos de cadeia ramificada. Uma maior quantidade de triptofano livre pode passar pela barreira, consequentemente, melhorando sua captação cerebral, que por sua vez, representa um maior aporte do precursor do neurotransmissor serotonina, que está relacionado com humor. ${ }^{33}$

Embora o descritor depressão não tenha se modificado, observou-se uma diminuição significativa nos escores indicativos de ansiedade estado no grupo que sofreu a intervenção. Esse resultado pode ser corroborado pelo aumento da qualidade de vida das voluntárias que foi constatado por melhora na capacidade física, vitalidade e, aspectos físicos e pelo aumento da média das dimensões medidas pelo SF-36. Acredita-se que esses resultados em relação à qualidade de vida também estão relacionados com a ausência de distorção da imagem corporal observada pelo BSQ, e a melhora da qualidade de sono.

Com esses resultados, observa-se que os aspectos psicobiológicos não se modificam de maneira isolada a partir de uma intervenção como esta. Embora melhoras efetivas na cognição não tenham sido alcançadas, outras variáveis se modificaram positivamente, como sono, atenção e cálculo e humor, o que leva a concluir que um programa de jogos pré-desportivos representa uma boa estratégia para melhorar aspectos psicobiológicos de idosas. Assim, práticas dessa natureza são desejáveis, pois seus resultados interferem na qualidade de vida dos idosos, não apenas por ser uma atividade de custo relativamente baixo, mas também por representar uma importante estratégia na configuração das políticas públicas com segmento de atuação nos 
idosos. O incentivo a essa prática certamente contribuiria para a mudança da sensibilidade brasileira em relação ao envelhecimento e para o modo como os indivíduos se preparam para as etapas mais avançadas da vida.

Os dados deste estudo referem-se a um período de intervenção de seis meses. Se o protocolo fosse realizado com uma duração maior, por exemplo, um ano, acredita-se que seria possível observar modificações mais expressivas nos aspectos psicobiológicos. Outra limitação deste estudo é a de que nenhum dos instrumentos utilizados analisa com precisão as mudanças fisiológicas decorrentes desse programa. Assim, mais estudos são necessários na investigação de práticas com caráter lúdicoesportivo para idosas.

\section{REFERÊNCIAS}

1. Instituto Brasileiro de Geografia e Estatística. Síntese de Indicadores Sociais: uma análise das condições de vida da população brasileira 2010. Estudos \& Pesquisas: informação demográfica e socioeconômica n. 27. Rio de Janeiro: IBGE; 2010 [acesso em 3 ago 2012]. Disponível: www.ibge.gov.br/home/estatistica/ populacao/condicaodevida/indicadoresminimos/ sinteseindicsociais2010/SIS_2010.pdf.

2. Pereira RJ, Cotta RMM, Franceschini SCC, Ribeiro RCL, Sampaio RF, Priore SE, et al. Contribuição dos domínios físico, social, psicológico e ambiental para a qualidade de vida global de idosos. Rev Psiquiatr 2006;28(1):27-38.

3. Dewsbury DA. "Psychobiology". Am Psychol 1991;46(3):198-205.

4. Geib LTC, Cataldo A Neto Neto, Wainberg R, Nunes ML. Sono e Envelhecimento. Rev Psiquiatr Bras 2003;25(3):453-65.

5. Gremeaux V, Gayda M, Lepers R, Sosner P, Juneau M, Nigam A. Exercise and longevity. Maturitas 2012;73(4):312-7.

6. Gáspari JC, Schwartz GM. O idoso e a ressignificação emocional do lazer. Psic: Teor e Pesq 2005;21(1):69-76.

\section{CONCLUSÃO}

Os resultados sugerem que um programa de seis meses de jogos pré-desportivos aplicado a idosos produz impacto positivo nos aspectos psicobiológicos. Por ser de baixo custo, sugere-se um maior empenho no incentivo dessa população para essa prática, uma vez que os benefícios refletem-se em uma boa qualidade de vida.

\section{AGRADECIMENTO}

Os autores são gratos a Everald Van Cooler por toda assistência técnica e científica, e ao suporte financeiro da AFIP, CEPE, CEMSA, CNPq, CAPES, CEPID/SONO-FAPESP (98/14303-3), Instituto do Sono, UNIFESP.
7. Bolonhini SZ, Paes RR. A proposta pedagógica do teaching games for understanding: reflexões sobre a iniciação esportiva. Pensar Prát 2009;12(2):1-9.

8. Huizinga J. Homo-ludens: o jogo como elemento da cultura. 2. ed. São Paulo: Perspectiva; 1990. 256 p.

9. Nóbrega ACL, Freitas EV, Oliveira MAB, Leitão MB, Lazzoli JK, Nahas RM, et al. Posicionamento oficial da Sociedade Brasileira de Medicina do Esporte e da Sociedade Brasileira de Geriatria e Gerontologia: atividade física e saúde no idoso. Rev Bras Med Esporte 1999;5(6):207-11.

10. Bunker D, Thorpe R. The curriculum model. In: R. Bunker D, Thorpe R, Almond L (Ed.). Rethinking games teaching. Loughborough: University of Technology; 1986. p. 7-10.

11. Brucki SMD, Nitrini R, Caramelli P, Bertolucci PHF, Okamoto IH. Sugestões para o uso do mini-exame do estado mental no Brasil. Arq Neuro-psiquiatr 2003;61(3B):777-81.

12. Biaggio AMB, Natalicio L. Manual para o inventário de ansiedade Traço-Estado (IDATE). Rio de Janeiro: Centro Editor de Psicologia Aplicada; 1979. 
13. Yesavage JA, Brink TL, Rose TL, Lum O, Huang V, Adey M, Leirer VO. Development and validation of a geriatric depression screening scale: a preliminary report. J Psychiat Res 1983;17(1):37-49.

14. Rohlfs ICPM, Rotta TM, Luft CB, Andrade A, Krebs RJ, Carvalho T. A Escala de Humor de Brunel (Brums): instrumento para detecção precoce da Síndrome do Excesso de Treinamento. Rev Bras Med Esporte 2008;14(3):176-81.

15. Ciconelli RM. Tradução para o portugues e validação do questionário genérico de avaliação de qualidade de vida "Medical outcome study 36-item short-form health survey (SF-36)" [dissertação de Mestrado]. São Paulo: Universidade Federal de São Paulo; 1997.

16. Bertolazi, AN. Tradução, adaptação cultural e validação de dois instrumentos de avaliação do sono: escala de sonolência de Epworth e Índice de qualidade de sono de Pittsburgh [dissertação de Mestrado]. Porto Alegre: Universidade Federal do Rio Grande do Sul; 2008.

17. Horne JA, Ostberg O. A self-assessment questionnaire to determine morningness-eveningness in human circadian rhythms. Int J Chronobiol 1976;4(2):97-110.

18. Associação Brasileira dos Institutos de Pesquisa de Mercado [ABIPEME]. Proposição para um novo critério de classificação sócio-econômica. São Paulo: Mímeo, 1978. 15 p.

19. Baecke JA, Burema J, Frijters JE. A short questionnaire for the measurement of habitual physical activity in epidemiological studies. Am J Clin Nutr 1982;36(5):936-42.

20. Cooper PJ, Taylor MJ, Cooper Z, Fairburn CG. The development and validation of the body shape questionnaire. Int J Eat Dis 1987;6(4):485-94.

21. Angevaren M, Vanhees L, Wendel-Vos W, Verhaar HJ, Aufdemkampe G, Aleman A, Verschuren WM. Intensity, but not duration, of physical activities is related to cognitive function. Eur J Cardiovasc Prev Rehabil 2007;14(6):825-30.

22. Antunes HKM, Santos RF, Heredia RAG, Bueno OFA, Mello MT. Alterações cognitivas em idosas decorrentes do exercício físico sistematizado. Revista da Sobama 2001;6(1):27-33.
23. Antunes HKM. A influência do exercício físico aeróbio em funções cognitivas e viscosidade do sangue de idosos normais [dissertação de de Mestrado]. São Paulo: Universidade Federal de São Paulo; 2003.

24. Rogers RL, Meyer JS, Mortel KF. After reaching retirement age physical activity sustains cerebral perfusion and cognition. J Am Geriatr Soc 1990;38(2):123-8.

25. Elwood PC, Pickering J, Bayer A, Gallacher JE. Vascular disease and cognitve function in older men in the Caerphilly cohort. Age Ageing 2002;31(1):43-8.

26. Meyer JS, Rogers RL, Judd BW, Mortel KF, Sims P. Cognition and cerebral blood flow fluctuate together in mult-infarct dementia. Stroke 1998;19(2):163-9.

27. Heyn P, Abreu BC, Ottenbacher KJ. The effects of exercise training on elderly persons with cognitive impairment and dementia: a meta-analysis. Arch Phys Med Rehabil 2004;85(10):1694-704.

28. Barnes JN, Taylor JL, Kluck BN, Johnson CP, Joyner MJ. Cerebrovascular reactivity is associated with maximal aerobic capacity in healthy older adults. J Appl Physiol (1985) 2013;114(10):1383-7.

29. Etnier JL, Nowell PM, Landers DM, Sibley BA. A meta-regression to examine the relationship between aerobic fitness and $\operatorname{cognitive~performance.~Brain~Res~}$ Rev 2006;52(1):119-30.

30. Erickson KI, Voss MW, Prakash RS, Basak C, Szabo A, Chaddock L, et al. Exercise training increases size of hippocampus and improves memory. Proc Natl Acad USA 2011;108(7):3017-22.

31. Blumenthal JA, Emery CF, Madden DJ, George LK, Coleman RE, Riddle MW, et al. Cardiovascular and behavioral effects of aerobic exercise training in healthy older men and women. J Gerontol 1989;44(5):147-57.

32. Antunes HK, Stella SG, Santos RF, Bueno OF, de Mello MT. Depression, anxiety and quality of life scores in seniors after endurance exercise program. Rev Bras Psiquiatr 2005;27(4):266-71.

33. Rossi L, Tirapegui J. Implicações do sistema serotoninérgico no exercício físico. Arq Bras Endocrinol Metab 2004;48(2):227-33. 